\title{
Understanding Neural Abstractive Summarization Models via Uncertainty
}

\author{
Jiacheng Xu Shrey Desai Greg Durrett \\ Department of Computer Science \\ The University of Texas at Austin \\ \{jcxu,gdurrett\}@cs.utexas.edu shreydesai@utexas.edu
}

\begin{abstract}
An advantage of seq2seq abstractive summarization models is that they generate text in a free-form manner, but this flexibility makes it difficult to interpret model behavior. In this work, we analyze summarization decoders in both blackbox and whitebox ways by studying on the entropy, or uncertainty, of the model's token-level predictions. For two strong pretrained models, PEGASUS (Zhang et al., 2020) and BART (Lewis et al., 2020) on two summarization datasets, we find a strong correlation between low prediction entropy and where the model copies tokens rather than generating novel text. The decoder's uncertainty also connects to factors like sentence position and syntactic distance between adjacent pairs of tokens, giving a sense of what factors make a context particularly selective for the model's next output token. Finally, we study the relationship of decoder uncertainty and attention behavior to understand how attention gives rise to these observed effects in the model. We show that uncertainty is a useful perspective for analyzing summarization and text generation models more broadly. ${ }^{1}$
\end{abstract}

\section{Introduction}

Recent progress in abstractive summarization has been fueled by the advent of large-scale Transformers pre-trained on autoregressive language modeling objectives (Hoang et al., 2019; Khandelwal et al., 2019; Lewis et al., 2020; Zhang et al., 2020). Despite their strong performance on automatic metrics like ROUGE (Lin, 2004), abstractive models are not as straightforward and interpretable as their extractive counterparts. Free-form generation in these models also leads to serious downstream errors, such as factual inconsistencies with the input document (Cao et al., 2018; Kryściński et al., 2020;

\footnotetext{
${ }^{1}$ Code is available at https://github.com/ jiacheng-xu/text-sum-uncertainty
}

Wang et al., 2020; Durmus et al., 2020; Goyal and Durrett, 2020). Although the interpretability of NLU models has been extensively studied (Ribeiro et al., 2016; Ghaeini et al., 2018; Jain and Wallace, 2019; Desai and Durrett, 2020), summarization models specifically have not received similar attention, with analysis efforts often focused on datasets and evaluation (Kryscinski et al., 2019).

In this work, we focus on interpreting and understanding abstractive summarization models through the lens of decoder uncertainty, or the entropy of decisions during generation. While uncertainty in generation has been studied from the perspective of data (Ott et al., 2018), sampling (Fan et al., 2018; Holtzman et al., 2019), and training (Correia et al., 2019; Kang and Hashimoto, 2020), it is underutilized as a technique for analysis and inspection of generation systems. We study two prominent summarization models, PEGASUS (Zhang et al., 2020) and BART (Lewis et al., 2020), fine-tuned on two English summarization datasets, CNN/Daily Mail (Hermann et al., 2015) and XSum (Narayan et al., 2018), to understand model behavior in each setting.

First, by comparing $n$-grams between the input document and generated summaries, we establish two coarse types for decoded tokens, copy and generate (See et al., 2017). We find that the entropy of the generation decision correlates with whether the model is copying or generating, as well as where in the sentence the token is. This paints a picture of certain contexts being more restrictive from the standpoint of generation, particularly early in sentences where a model has not "decided" what to copy yet, and illustrates the interaction of content selection and lexical choice. Second, we extend this analysis by looking at how uncertainty relates to the syntax of the generated sentence: whether uncertainty connects to syntactic notions of surprisal (Roark et al., 2009) and how the entropy 
varies across certain syntactic productions. Finally, we derive a way to quantify decoder attention by aggregating distinct self-attention heads, revealing the correlation between the attention entropy and prediction entropy, and investigating the correspondence between the prediction entropy and the fraction of the past and future decoded tokens.

Taking this analysis together, we find that the abstractiveness of reference summaries fundamentally changes model behavior: the extractive nature of CNN/DM makes most of its decisions low entropy and copy-oriented while the model maintains higher uncertainty on XSum, yielding more abstractive summaries. More broadly, we show that uncertainty is a simple but effective tool to characterize decoder behavior in text generation.

\section{Model and Experimental Setup}

Our experiments use PEGASUS (Zhang et al., 2020) and BART (Lewis et al., 2020), two state-ofthe-art seq2seq pre-trained models. We use the large version of these two models, which have 16 and 12 Transformer layers, respectively. Both models have pre-training objectives tailored somewhat to this problem domain: seq2seq modeling for denoising (BART) or infilling of masked-out sentences (PEGASUS). We directly use the pre-trained models from Wolf et al. (2019). ${ }^{2}$

As reported in the original papers and measured by ROUGE-1/2/L (Lin, 2004), PEGASUS achieves $44.17 / 21.47 / 41.11$ on CNN/DM (Hermann et al., 2015) and 47.21/24.56/39.25 on XSum (Narayan et al., 2018), and BART achieves $44.16 / 21.28 / 40.90$ and 45.14/22.27/37.25.

Entropy. Entropy is a standard measure of uncertainty in a probabilistic distribution. Given a discrete random variable $X$ with all possible outcomes $x_{1}, \cdots, x_{n}$, the entropy of $X$ is defined as $H(X)=-\sum_{i=1}^{n} P\left(x_{i}\right) \log P\left(x_{i}\right)$.

For pre-trained Transformers, the domain of the predictions (the vocabulary) is large and also differs between models. The vocabulary sizes for PEGASUS and BART are 96,103 and $50,265,{ }^{3}$ and the prediction distribution is usually long-tailed.

\footnotetext{
${ }^{2}$ Specifically, google/pegasus-cnn_dailymail, google/pegasus-xsm, facebook/bart-large-cnn, and facebook/bart-large-xsum for PEGASUS and BART on these two datasets.

${ }^{3}$ Note that entropy generally increases as the variable's domain grows: a uniform distribution over 10,000 outcomes has entropy 9.21, while a uniform distribution over 100,000 outcomes has entropy 11.51 .
}

To combat this, nucleus sampling (Holtzman et al., 2019) is used to sample from only the top $1-p$ most probable outcomes (the nucleus) to avoid generating very unlikely tokens. To more fairly compare models with different vocabulary sizes, and to better reflect the actual sampling distribution, we therefore compute all entropy values in this work over the nucleus distribution. That is, we sort the prediction distribution $P\left(x_{i}\right)$ in descending order and get a minimal set of tokens where $V^{\min }=\left\{x \mid \sum_{x_{i} \in V^{\min }} P\left(x_{i}\right) \geq p\right\}$. Then we renormalize the distribution as follows:

$$
P^{\prime}\left(x_{i}\right)= \begin{cases}\frac{P\left(x_{i}\right)}{p^{\prime}} & \text { if } x_{i} \in V^{\mathrm{min}} \\ 0 & \text { otherwise. }\end{cases}
$$

where the cumulative probability $p^{\prime}=$ $\sum_{x_{i} \in V^{\min }} P\left(x_{i}\right)$. We use $p=0.95$ for all experiments. The entropy $H(X)$ is computed based on the new distribution $P^{\prime}\left(x_{i}\right)$.

\section{Model Uncertainty during Generation}

In this section, we analyze and compare the prediction uncertainty from different models and different datasets by inspecting entropy values during generation, allowing us to localize uncertainty to certain positions in a decoded sentence. A principle factor that past work has investigated is the amount of copying in abstractive summarization models (See et al., 2017; Paulus et al., 2018). We first aim to understand how decisions to copy document content or generate new text are reflected in the model's uncertainty.

One complicating factor is that while BART and PEGASUS both exhibit a mix of copying and novel generation, they do not have an explicit copy operation like in past models and so these behaviors are more difficult to define. We first separate generation decisions by bigrams that appear in the input document (existing bigrams) or whether they are free-form generations (novel bigrams). ${ }^{4}$

Figure 1 shows a histogram of model entropies broken down by these two categories. Most notably, there is a strong correlation between copy-like behavior and the entropy of the model's prediction distribution. On CNN/DM, we see that low entropy decisions are largely those generating existing bigrams, and conversely, existing bigrams are usually generated with low entropy. New bigrams are generated with a broad range of high

\footnotetext{
${ }^{4}$ Bigrams are defined based on tokens rather than wordpieces, and so may consist of more than two generation steps.
} 


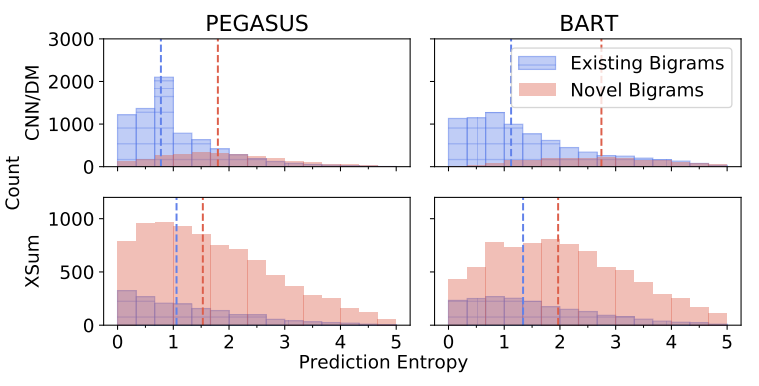

Figure 1: Next token entropies computed on 10K generation steps from PEGASUS CNN/DM $_{\text {PEGASUS }}$ XSUM,

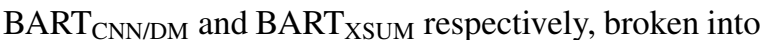
two cases: an Existing Bigram means the bigram just generated occurs in the input document, while a Novel Bigram is an organic model generation. These cases are associated with low entropy and high entropy actions, respectively. The $\mathrm{x}$-axis shows the entropy (truncated at 5), and the y-axis shows the count of bigram falling in each bin. The dashed lines indicate the median of each distribution.

entropy values, and are much more frequent on XSum. These results align with our manual analysis of these summaries: PEGASUS ${ }_{\mathrm{CNN} / \mathrm{DM}}$ and BART $_{\text {CNN/DM }}$ summaries largely consist of spans from the input document with minor compression while PEGASUS XSUM $_{\text {and }}$ BART $_{X S U M}$ summaries involve stitching together disparate concepts and paraphrasing key details. This reflects a corresponding divergence in the gold summaries, where CNN/DM summaries are far more extractive than those in XSum.

Critically, though the entropy distributions are dissimilar across the two datasets, we see regularities among the approximate copy and generate operations: on CNN/DM and XSum, the median entropy values of using existing bigrams are 0.95 and 1.20 , respectively, and for generating new bigrams, 2.27 and 1.75 .

With this connection between entropy and copying behavior, we make the following additional observations based on Figures 1 and 2:

Entropy varies across token positions, especially on CNN/DM. In Figure 2, we depict a different view of entropy, looking at the decoding process as it progresses through each sentence. Across both CNN/DM and XSum, models are most uncertain at the beginning of the sentence and least uncertain at the end of the sentence. However, the rate at which entropy drops off is quite different: on CNN/DM, the entropy after decoding $20 \%$ of tokens falls below 2, while the entropies

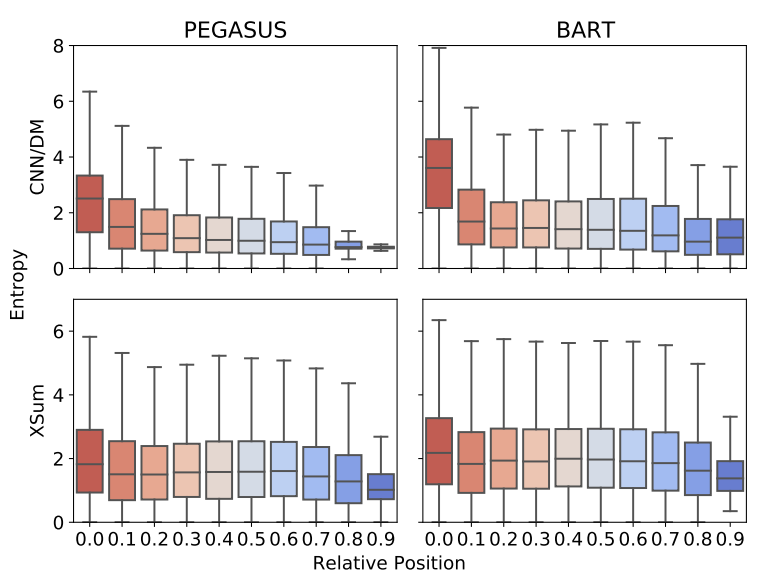

Figure 2: Prediction entropy values by relative sentence positions. For example, 0.0 indicates the first $10 \%$ of tokens in a sentence, and 0.9 is the last $10 \%$ of tokens. PEGASUS $_{\mathrm{CNN} / \mathrm{DM}}$ and $\mathrm{BART}_{\mathrm{CNN} / \mathrm{DM}}$ make highly uncertain decisions to start, but then entropy decreases, suggesting that these models may be copying based on a sentence prefix. Entropies on XSum are more constant across the sentence.

on XSum only begin to considerably drop after decoding $80 \%$ of tokens. Our manual analysis suggests the following characterization: to generate each sentence on CNN/DM, the model makes some high-entropy decisions to identify a sentence and begin to copy its prefix, followed by a series of low entropy decisions to copy that sentence's content. On XSum, which is highly abstractive and features single sentence summaries, content planning and generation are less clearly decoupled.

PEGASUS copies and generates more tokens with entropy $<1$. BART and PEGASUS report similar ROUGE results on CNN/DM, but these models do not place the same distributions over summaries. PEGASUS has more low-entropy copying decisions, and its start-of-sentence entropies are also significantly lower (Figure 2). This suggests that it is more confident than BART in selecting content to discuss next. There are also more low-entropy generation decisions, particularly on XSum.

\section{Entropies of Syntactic Productions}

Having observed connections between sentence position and entropy, we now flesh out this analysis from the lens of syntax, focusing in particular on uncertainty at constituent boundaries. From our PEGASUS generations on CNN/DM and XSum, 

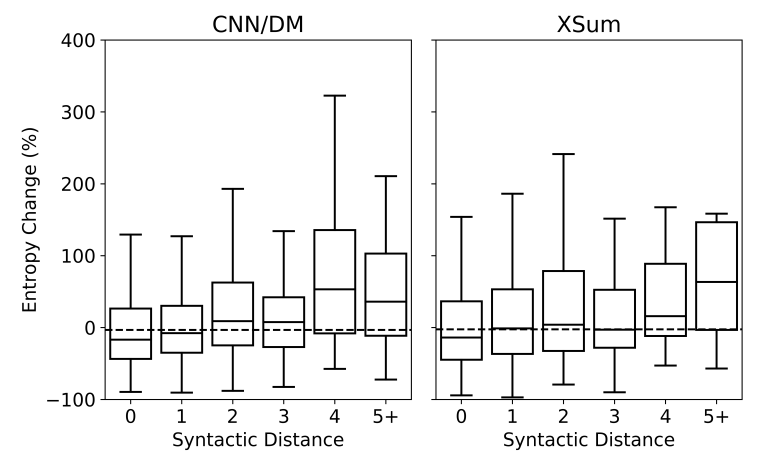

Figure 3: Correlating syntactic distance between neighboring tokens with the entropy change in those tokens' generation decisions for PEGASUS summaries. The median entropy change is depicted as a dashed black line. At points of high syntactic distance, the model's behavior is less restricted by the context, correlating with higher entropy.

we obtain constituency parses for each summary sentence using the Berkeley Neural Parser (Kitaev and Klein, 2018) and explore connections between syntax and uncertainty in more depth.

Low and high entropy decisions can be localized to constituent span boundaries. Parsing has long been used to explain psycholinguistic notions of surprisal (Hale, 2001; Roark et al., 2009, inter alia), which are in turn related to uncertainty under a language model. In our case, uncertainty about generating a text is a different notion than uncertainty when a reader is processing it. Hence, rather than looking at an incremental parser's behavior, we instead look at a simpler notion of syntactic distance (Shen et al., 2018), or the number of left and right parentheses between $w_{t}$ and $w_{t+1}$ in a linearized constituency tree. Our hypothesis is that when these words exhibit high syntactic distance, this word boundary is a "choice point" where the model may be less restricted in what it can choose to generate next.

Figure 3 shows the correlation between syntactic distance and the percent change in entropy between the adjacent tokens. On both CNN/DM and XSum, we see two patterns emerge: generating a token within the same immediate parent constituent (i.e., zero syntactic distance) is typically a certain decision, while generating a token belonging to a new constituent is an increasingly uncertain decision. From these results, we can draw a parallel to the copy vs. generate behavior established in Section 3; for example, generating York after New

\begin{tabular}{ll}
\hline Production Rule & Example \\
\hline $\mathrm{NP} \rightarrow \mathrm{NP}: \mathrm{NP}$ & $\begin{array}{l}{[\text { Arsenal vs Reading }]_{1.2}[:]_{0.6}} \\
{[\text { the game that changed the }} \\
\text { game }]_{3.1}\end{array}$ \\
$\mathrm{NP} \rightarrow \mathrm{NP}, \mathrm{SBAR}$, & $\begin{array}{l}\text { [driver }]_{0.5}[]_{0.4}[\text { who has not } \\
\text { been identified }]_{2.2}[,]_{0.1}\end{array}$ \\
\hline $\mathrm{NP} \rightarrow \mathrm{CD} \mathrm{NN} \mathrm{NNS}$ & $\begin{array}{l}{[16]_{0.07}[\text { felony }]_{0.05}[\text { counts }]_{0.01}} \\
{[\text { April }]_{0.04}[3]_{0.1}}\end{array}$ \\
\hline $\mathrm{NP} \rightarrow \mathrm{NNP} \mathrm{CD}$ &
\end{tabular}

Table 1: Examples of specific NP productions with high entropy (top) and low entropy (bottom). The notation $[\mathrm{Y}]_{H(Y)}$ implies the constituent $Y$ is generated with entropy $H(Y)$.

might be straightforward, perhaps due to a direct copy from the document, but generating a prepositional phrase might be more challenging due to the large search space of possible constructions or the higher chance that the model might delete this constituent.

Low entropy spans are often short, specific units of information. We also investigate the average entropy of spans within a rule production to uncover what types of spans are likely to elicit certainty or uncertainty during generation. In Table 1 , we see qualitatively that productions with low average entropy productions are short extracts of document content, such as 16 felony counts. These are largely factual, often containing cardinal values, and more likely to be copied. Within these constituents, the model is very certain about what to generate next, supporting the connection with low syntactic distance.

\section{Understanding Decoder Self-Attention}

While we have analyzed the model's predictions, we have not yet determined how the different behaviors we see emerge from the context. Our goal is to explore what the encoder attention places its emphasis during generation and how it correlates with the prediction entropy. ${ }^{5}$

Blocking Low-information Tokens. Analyzing the inner workings of attention in Transformers is challenging (Clark et al., 2019; Kovaleva et al., 2019), particularly because many heads are useless, redundant, or noisy, and they frequently attend to

\footnotetext{
${ }^{5}$ In PEGASUS and BART models, the encoder and decoder attention during decoding are two separate distributions where the encoder attention looks at the encoding context and the decoder attention attends to the previously decoded tokens. In this paper we chiefly examine the encoder attention to understand how the model references the input document.
} 


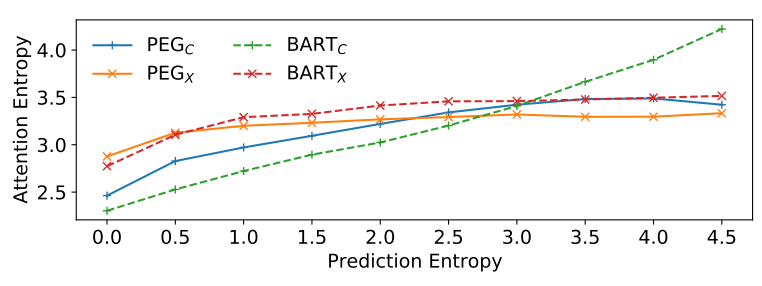

Figure 4: Correlation between attention entropy and prediction entropy of PEG(ASUS) and BART on $\mathrm{C}(\mathrm{NN} / \mathrm{DM})$ and $\mathrm{X}(\mathrm{Sum})$. We compute the mean value of the attention entropy within each bucket of prediction entropy. The uncertainty of attention strongly correlates with the entropy of the model's prediction.

low-information tokens such as end-of-sentence markers or periods. Inspired by tf-idf (Joachims, 1997), we propose a method to compute a set of tokens most meaningfully attended to by the model. If a token in the encoding document is attended to across many time steps (like a word appearing in many documents in tf-idf), we want to disregard it in our analysis.

Let $T$ denote the number of decoder timesteps and $L$ be the length of the source document. We compute an aggregate attention matrix $S \in \mathbb{R}^{T \times L}$ by summing the attentions across all heads and all layers. We then compute a count of how often each token is attended to above a threshold $q$ : $f_{l}=$ $\sum_{t=1}^{T}\left[\mathbb{1}\left(s_{t l} \geq q\right)\right]$ and discard the attention values on tokens with the highest $f$ score. In practice we discard $5 \%$ of tokens from the source document.

Attention Entropy. One natural question we can ask is whether there is a connection between entropy of the attention distribution and entropy of the decoder's prediction. This relationship is shown in Figure 4, where each point represents the mean attention entropy within the corresponding prediction entropy bucket. The attention entropy is especially low where the prediction entropy ranges from 0 to 0.5 . For cases with prediction entropy greater than 1.5 , the attention entropy saturates and no longer grows with the prediction entropy except the $\mathrm{BART}_{\mathrm{CNN} / \mathrm{DM}}$. While attention entropy is probably not "causing" the low decoder entropy per se, nevertheless decoder entropy provides a lens into the inner workings of the Transformer model.

Projecting Attention to Vocabulary. We hypothesize that low decoder entropies may arise if the model is heavily attending to certain relevant tokens, particularly the (about to be predicted) token $y_{t}$ of time step $t$ and the input token of this time

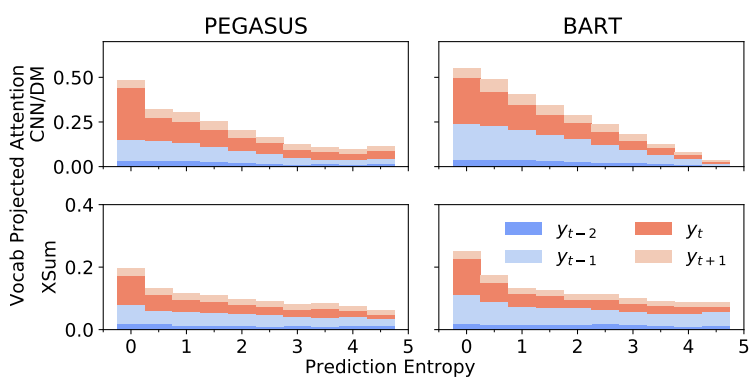

Figure 5: Vocabulary projected attention attending to the last input $y_{t-2}$, current input $y_{t-1}$, current output $y_{t}$, and next output $y_{t+1}$. When the prediction entropy is low, the attention mostly focus a few tokens including the current input $y_{t-1}$ and current output $y_{t}$.

step $x_{t}$, equivalent to $y_{t-1}$. For the predicted token $y_{t}$, we compute the vocabulary projected attention value $\sum_{l=1}^{L} \mathbb{1}\left[\operatorname{token}_{l}=y_{t}\right] s_{t l}$ where we accumulate the attention of all of the occurrences of the specified token $y_{t}$ in the document. The higher the value, the more attention put to the encoding token(s) which are predicted for this time step during decoding. We can define the value for last time step input $y_{t-2}$, current time step input $y_{t-1}$, and the not-yet-decoded token $y_{t+1}$ for next time step.

We show the relationship between the vocabulary projected attention and the prediction entropy in Figure 5. Visualizations for both models and both datasets show that when the prediction entropy is low, the attention focuses heavily on a few tokens including the current input token and the current token to predict. This suggests a potential mechanism where the model indexes into the source document by attending to $y_{t-1}$, then strongly identifies and "reads off" $y_{t}$ as the next token to generate.

\section{Conclusion}

This work analyzes pre-trained summarization models via uncertainty, or the entropy of decoding decisions. We pursue several lines of inquiry: uncertainty can help us understand copying document spans vs. generating novel text, the behavior of models in different syntactic environments, and coarse properties of the model's attention distribution. All of these give insight into what conditions most heavily restrict the model's generation: generating an observed bigram (copying), low syntactic distance, and attention which can easily identify decoder context in the source document. We believe this approach can power future analyses of pre-trained text generation systems. 


\section{Acknowledgments}

This work was partially supported by NSF Grant IIS-1814522, NSF Grant SHF-1762299, a gift from Salesforce Inc, and an equipment grant from NVIDIA. The authors acknowledge the Texas Advanced Computing Center (TACC) at The University of Texas at Austin for providing HPC resources used to conduct this research. Results presented in this paper were obtained using the Chameleon testbed supported by the National Science Foundation. Thanks as well to the anonymous reviewers for their helpful comments.

\section{References}

Ziqiang Cao, Furu Wei, Wenjie Li, and Sujian Li. 2018. Faithful to the Original: Fact Aware Neural Abstractive Summarization. In Proceedings of the AAAI Conference on Artificial Intelligence (AAAI).

Kevin Clark, Urvashi Khandelwal, Omer Levy, and Christopher D. Manning. 2019. What Does BERT Look at? An Analysis of BERT's Attention. In Proceedings of the ACL Workshop BlackboxNLP: Analyzing and Interpreting Neural Networks for NLP.

Gonçalo M Correia, Vlad Niculae, and André FT Martins. 2019. Adaptively sparse transformers. In Proceedings of the 2019 Conference on Empirical Methods in Natural Language Processing and the 9th International Joint Conference on Natural Language Processing (EMNLP-IJCNLP), pages 2174-2184.

Shrey Desai and Greg Durrett. 2020. Calibration of Pre-trained Transformers. In Proceedings of the Conference on Empirical Methods in Natural Language Processing (EMNLP).

Esin Durmus, He He, and Mona Diab. 2020. FEQA: A Question Answering Evaluation Framework for Faithfulness Assessment in Abstractive Summarization. In Proceedings of the Annual Conference of the Association for Computational Linguistics (ACL).

Angela Fan, Mike Lewis, and Yann Dauphin. 2018. Hierarchical Neural Story Generation. In Proceedings of the Annual Conference of the Association for Computational Linguistics (ACL).

Reza Ghaeini, Xioali Fern, and Prasad Tadepalli. 2018. Interpreting Recurrent and Attention-Based Neural Models: a Case Study on Natural Language Inference. In Proceedings of the Conference on Empirical Methods in Natural Language Processing (EMNLP).

Tanya Goyal and Greg Durrett. 2020. Evaluating Factuality in Generation with Dependency-level Entailment. In Findings of the Conference on Empirical Methods in Natural Language Processing (Findings of EMNLP).
John Hale. 2001. A probabilistic Earley parser as a psycholinguistic model. In Proceedings of the Second Meeting of the North American Chapter of the Association for Computational Linguistics.

Karl Moritz Hermann, Tomás Kočiský, Edward Grefenstette, Lasse Espeholt, Will Kay, Mustafa Suleyman, and Phil Blunsom. 2015. Teaching Machines to Read and Comprehend. In Proceedings of the Conference on Neural Information Processing Systems (NeurIPS).

Andrew Pau Hoang, Antoine Bosselut, Asli Çelikyilmaz, and Yejin Choi. 2019. Efficient Adaptation of Pretrained Transformers for Abstractive Summarization. arXiv preprint arXiv:1906.00138.

Ari Holtzman, Jan Buys, Li Du, Maxwell Forbes, and Yejin Choi. 2019. The Curious Case of Neural Text Degeneration. In Proceedings of the Conference on International Conference on Learning Representations (ICLR).

Sarthak Jain and Byron C. Wallace. 2019. Attention is not Explanation. In Proceedings of the 2019 Conference of the North American Chapter of the Association for Computational Linguistics: Human Language Technologies (NAACL-HLT).

Thorsten Joachims. 1997. A Probabilistic Analysis of the Rocchio Algorithm with TFIDF for Text Categorization. In ICML.

Daniel Kang and Tatsunori Hashimoto. 2020. Improved natural language generation via loss truncation. arXiv preprint arXiv:2004.14589.

Urvashi Khandelwal, K. Clark, Daniel Jurafsky, and Lukasz Kaiser. 2019. Sample Efficient Text Summarization Using a Single Pre-Trained Transformer. arXiv preprint arXiv:1905.08836.

Nikita Kitaev and Dan Klein. 2018. Constituency Parsing with a Self-Attentive Encoder. In Proceedings of the Annual Meeting of the Association for Computational Linguistics (ACL).

Olga Kovaleva, Alexey Romanov, Anna Rogers, and Anna Rumshisky. 2019. Revealing the Dark Secrets of BERT. In Proceedings of the Conference on Empirical Methods in Natural Language Processing and the International Joint Conference on Natural Language Processing (EMNLP-IJCNLP).

Wojciech Kryscinski, Nitish Shirish Keskar, Bryan McCann, Caiming Xiong, and Richard Socher. 2019. Neural Text Summarization: A Critical Evaluation. In Proceedings of the 2019 Conference on Empirical Methods in Natural Language Processing and the 9th International Joint Conference on Natural Language Processing (EMNLP-IJCNLP).

Wojciech Kryściński, Bryan McCann, Caiming Xiong, and Richard Socher. 2020. Evaluating the Factual Consistency of Abstractive Text Summarization. In Proceedings of the Conference on Empirical Methods in Natural Language Processing (EMNLP). 
Mike Lewis, Yinhan Liu, Naman Goyal, Marjan Ghazvininejad, Abdelrahman Mohamed, Omer Levy, Veselin Stoyanov, and Luke Zettlemoyer. 2020. BART: Denoising sequence-to-sequence pretraining for natural language generation, translation, and comprehension. In Proceedings of the 58th Annual Meeting of the Association for Computational Linguistics, pages 7871-7880, Online. Association for Computational Linguistics.

Chin-Yew Lin. 2004. ROUGE: A Package for Automatic Evaluation of Summaries. In Proceedings of the Annual Meeting of the Association for Computational Linguistics (ACL).

Shashi Narayan, Shay B. Cohen, and Mirella Lapata. 2018. Don't Give Me the Details, Just the Summary! Topic-Aware Convolutional Neural Networks for Extreme Summarization. In Proceedings of the Conference on Empirical Methods in Natural Language Processing (EMNLP).

Myle Ott, Michael Auli, David Grangier, and Marc'Aurelio Ranzato. 2018. Analyzing uncertainty in neural machine translation. In International Conference on Machine Learning, pages 3956-3965.

Romain Paulus, Caiming Xiong, and Richard Socher. 2018. A Deep Reinforced Model for Abstractive Summarization. In Proceedings of the International Conference on Learning Representations (ICLR).

Marco Tulio Ribeiro, Sameer Singh, and Carlos Guestrin. 2016. "Why Should I Trust You?": Explaining the Predictions of Any Classifier. In Proceedings of the ACM SIGKDD Conference on Knowledge Discovery and Data Mining (SIGKDD).

Brian Roark, Asaf Bachrach, Carlos Cardenas, and Christophe Pallier. 2009. Deriving lexical and syntactic expectation-based measures for psycholinguistic modeling via incremental top-down parsing. In Proceedings of the 2009 Conference on Empirical Methods in Natural Language Processing, pages 324-333, Singapore. Association for Computational Linguistics.

Abigail See, Peter J. Liiu, and Christopher D. Manning. 2017. Get To The Point: Summarization with Pointer-Generator Networks. In Proceedings of the Annual Meeting of the Association for Computational Linguistics (ACL).

Yikang Shen, Zhouhan Lin, Chin wei Huang, and Aaron Courville. 2018. Neural Language Modeling by Jointly Learning Syntax and Lexicon. In Proceedings of the International Conference on Learning Representations (ICLR).

Alex Wang, Kyunghyun Cho, and Mike Lewis. 2020. Asking and Answering Questions to Evaluate the Factual Consistency of Summaries. In Proceedings of the Annual Conference of the Association for Computational Linguistics (ACL).
Thomas Wolf, Lysandre Debut, Victor Sanh, Julien Chaumond, Clement Delangue, Anthony Moi, Pierric Cistac, Tim Rault, Rémi Louf, Morgan Funtowicz, and Jamie Brew. 2019. HuggingFace's Transformers: State-of-the-art Natural Language Processing. arXiv preprint arXiv:1910.03771.

Jingqing Zhang, Yao Zhao, Mohammad Saleh, and Peter J. Liu. 2020. PEGASUS: Pre-training with Extracted Gap-sentences for Abstractive Summarization. Proceedings of Machine Learning Research. PMLR. 